\title{
TERMORREGULACIÓN EN VERTEBRADOS TERRESTRES COMO CONCEPTO INTEGRADOR EXPLÍCITO EN LA ENSEÑANZA DE LAS CIENCIAS NATURALES
}

\author{
ERIKA PATRICIA DAZA-PÉREZ II"* \\ https://orcid.org/0000-0002-7549-9198 \\ CHARBEL NIÑO EL-HANI III IN** \\ https://orcid.org/0000-0002-2308-3983
}

RESUMEN: El artículo presenta un análisis del tratamiento de termorregulación en la literatura sobre didáctica de las ciencias naturales, en algunos libros de texto de Colombia y en los documentos oficiales que reúnen orientaciones para la enseñanza y evaluación en ciencias de este mismo país. A partir de ello pone de manifiesto que termorregulación no constituye una temática central en la enseñanza de la biología y es citado sin conexión con otras temáticas. Discute algunos fundamentos para una enseñanza integrada y explícita de termorregulación en conexión con otras temáticas centrales en la enseñanza de la biología y propone un diagrama que constituye un modelo didáctico para abordar termorregulación como un mecanismo homeostático.

Palabras clave: Termorregulación. Homeóstasis. Mecanismos biológicos.

\section{TERMORREGULAÇÃO EM VERTEBRADOS TERRESTRES COMO CONCEITO INTEGRADOR EXPLÍCITO NO ENSINO DAS CIÊNCIAS NATURAIS}

RESUMO: O artigo apresenta uma análise do tratamento da termorregulação na literatura sobre didática das ciências naturais, em alguns livros didáticos da Colômbia e nos documentos oficiais que reúnem diretrizes para o ensino e a avaliação em ciências desse mesmo país. A partir disso, mostra que a termorregulação não é tratada como uma questão central no ensino da biologia, sendo citada sem conexão com outros tópicos. São discutidos alguns fundamentos para um

\author{
*Doutora em Ensino, Filosofia e \\ História das Ciências (PPGEFHC) \\ -UFBA/UEFS (Brasil). Magíster \\ en Docencia de la Química UPN \\ (Colombia). Licenciada en Biología y \\ Química, UPTC (Colombia). \\ Coordinadora Académica Colegio \\ Integrado Divino Niño, Capitanejo, \\ Santander-Colombia. Investigadora \\ Laboratório de Ensino, Filosofia e \\ História da Biologia (LEFHBio). \\ E-mail:<erdaza1212@yahoo.es > \\ * *Doutor em Educação (USP, 2000), \\ Mestre em Educação (UFBA, 1996) \\ e Bacharel em Ciências \\ Biológicas (UFBA, 1992). \\ E-mail:<charbel.elhani@gmail.com
}

\footnotetext{
I Universidade Federal da Bahia, Laboratório de Ensino, Filosofia e História da Biologia, Salvador, BA - Brasil.

II Colegio Integrado Divino Niño, Capitanejo, Santander - Colombia.

III Universidade Federal da Bahia, Instituto de Biologia, Salvador, BA - Brasil.

IV Universidade Federal da Bahia, Instituto Nacional em Estudos Interdisciplinares e Transdisciplinares em Ecologia e Evolução, Salvador, BA - Brasil.
} 
ensino integrado e explícito sobre termorregulação, em conexão com outros tópicos centrais no ensino de biologia, e propõe-se um diagrama que constitui um modelo didático para abordar a termorregulação como um mecanismo homeostático.

Palavras-chave: Termorregulação. Homeostase. Mecanismos biológicos.

\section{THERMOREGULATION IN TERRESTRIAL VERTEBRATES AS EXPLICIT INTEGRATING CONCEPT IN THE NATURAL SCIENCES TEACHING}

ABSTRACT: The article presents an analysis of the treatment of thermoregulation in the literature on natural sciences teaching, in some textbooks from Colombia and in official documents that present guidelines for science teaching and assessment in this country. This analysis shows that thermoregulation is not taken as a central issue in biology teaching and is cited without connection with other topics. We discuss some grounds for an integrated and explicit teaching on thermoregulation, in connection with other central topics in biology teaching, and propose a diagram that constitutes a didactics model to address thermoregulation in the classroom, as a homeostatic mechanism.

Keywords: Thermoregulation. Homeostasis. Biological mechanisms. 


\section{INTRODUCCIÓN}

Uno de los desafíos en la enseñanza y el aprendizaje de la Biología es superar visiones aisladas y fragmentadas de fenómenos y, así, entender que los mecanismos individuales son parte de fenómenos globales (TRUJILLO et al. 2015), alcanzando una comprensión integral (VERHOEFF et al., 2008), coherente con perspectivas sistémicas (SVOBODA \& PASSMORE, 2013)

En los estudiantes, existe una tendencia a explicar los fenómenos complejos como una serie de fenómenos no relacionados que comparten algunos principios subyacentes (MODELL 2000), mediante reglas simples, o como fenómenos simples, apelando al razonamiento causal lineal como si se tratara de cadenas secuenciales de causas y efectos, y no de patrones sistémicos, de relaciones causales más intrincadas, es decir, a menudo si pierde de vista una causalidad compleja (GROTZER, 2003). Por ejemplo, resulta difícil comprender la homeostasis porque varios mecanismos y submecanismos implicados no son percibidos mediante la observación de sus manifestaciones, sino que involucran una apreciación dinámica (JUNGWIRTH \& DREYFUS, 1992; WESTBROOK \& MAREK, 1992; ASSARAF et al., 2013) e interdisciplinaria (ÇIMER 2012). Particularmente, en la regulación de la temperatura, los estudiantes identifican las consecuencias y respuestas ante la pérdida de la estabilidad, es decir, la razón próxima que conduce al cambio, pero ignoran los procesos fisiológicos que producen el fenómeno (BUDDING 1996; ASSARAF et al., 2013)

Desde esa perspectiva, termorregulación constituye un concepto de gran interés didáctico en la educación básica secundaria como contexto para explicar la homeóstasis y establecer relaciones con conceptos definidos en el currículo de la educación básica que son fundamentales en el aprendizaje de las Ciencias Naturales (por ejemplo, adaptación, evolución, mecanismos de intercambio de energía, calor, temperatura etc.). Esto es el caso en Colombia (MEN, estándares básicos de competencia) así como en otros países (NCR 2009). Por lo anterior, el presente artículo se propone desarrollar argumentos para que la termorregulación en animales sea considerada como una temática integradora en la enseñanza de la biología, al contrario de prácticas de enseñanza que la reducen a un concepto secundario, apenas citado en relación con otros conceptos.

Presentamos un análisis del tratamiento de termorregulación en la literatura sobre didáctica de las ciencias naturales, en algunos libros de texto de Colombia y en los documentos oficiales que reúnen orientaciones para la enseñanza y evaluación en ciencias de este mismo país. Por último, discutimos algunos fundamentos para una enseñanza integrada y explícita de termorregulación en animales vertebrados terrestres.

\section{MÉTODOS}

El abordaje metodológico estuvo constituido por dos fases: La primera comprendió la revisión de literatura y consulta a profesores de básica secundaria, mientras que la segunda un análisis la información recolectada y la construcción de una propuesta didáctica. 


\section{Primera fase}

Etapa 1. Revisión de literatura: Revisamos hasta el 29 de marzo de 2018 la literatura en didáctica de las ciencias sobre termorregulación y homeóstasis sin delimitar un periodo de años. Consultamos las bases de datos ERIC, Scielo, Redalyc, Dialnet, Latindex y Google Acadêmico, sin delimitar año de publicación y usando los términos de búsqueda 'thermoregulation', 'temperature regulation', "thermal regulation", 'termorregulación', 'regulación de la temperatura', 'termorregulação', 'regulação da temperatura', 'homeostasis' 'homeóstasis' y 'homeostase'.

Etapa 2. Consulta a profesores: Aplicamos un cuestionario de cinco preguntas mediante el aplicativo Google Forms a 16 profesores de Ciencias Naturales de 11 municipios de Colombia en el nivel de básica secundaria. Indagaba por el lugar de trabajo, si ellos enseñaban termorregulación en animales, el grado en el cual lo enseñaban, los conceptos con los cuales lo asociaban, y los libros de texto que empleaban en sus clases. El cuestionario fue divulgado a través de mensaje de correo a docentes de colegios públicos, grupos de WhatsApp ${ }^{\circledR}$ y Facebook ${ }^{\circledR}$.

Etapa 3. Análisis de documentos curriculares y libros de texto: Tambiénanalizamos los Estándares Básicos de Competencias y los Derechos Básicos de Aprendizaje (DBA) para Ciencias Naturales propuestos por el Ministerio de Educación Nacional de Colombia (MEN), así como documentos del Instituto Colombiano para el Fomento de la Educación Superior - ICFES sobre las Pruebas Saber ${ }^{1}$ Grados 3, 5, 9 y 11. Analizamos también algunos libros de texto usados por los profesores que respondieron el cuestionario.

Se identificaron los grados y estándares que citan o tienen relación con el concepto de termorregulación, así como la secuencia de temas, para sugerir el mejor grado para enseñar el concepto, mientras que en los libros identificamos si el concepto era abordado, las temáticas donde si hacía referencia al mismo y algunas observaciones generales sobre su tratamiento.

\section{Segunda fase}

\section{Análisis de la información recolectada y construcción de propuesta didáctica.}

Con base en esa revisión, en la segunda fase identificamos relaciones con otros conceptos, grados y espacios en la secuenciación curricular que juzgamos de mayor pertinencia para abordar el tema. También analizamos el fenómeno desde la perspectiva de la modelación de mecanismos, para reconocer componentes e interacciones, estructurando un diagrama que provee directrices para la enseñanza sobre termorregulación. El diagrama sigue el modelo simplificado de homeóstasis propuesto por Modell et al. (2015) fue sometido a evaluación por tres expertos en fisiología animal y humana, para su validación por pares, y socializado en dos eventos académicos. Tanto los expertos como la audiencia en los eventos hicieran sugerencias de ajustes, los cuales fueron hechos al diagrama. 


\section{TERMORREGULACIÓN - CONCEPTO NO EXPLICITO}

En este apartado se dilucida sobre el tratamiento de termorregulación en animales en la literatura en didáctica de las Ciencias Naturales, en orientaciones curriculares de Colombia, en algunos libros de texto y en comentarios de profesores de Ciencias Naturales en la Educación Básica respecto de la enseñanza del concepto.

\section{Literatura en didáctica de las Ciencias Naturales.}

La mayoría de los estudios encontrados que abordan termorregulación en el contexto didáctico están focalizados en la educación superior. Por ejemplo, el módulo instruccional propuesto por Stevenson en 1979 señala las limitaciones de los modelos puramente físicos de equilibrio térmico en los animales puesto que, según las condiciones del ambiente físico y sus características fisiológicas, estos organismos pueden emplear también mecanismos comportamentales, fisiológicos y morfológicos e integrar algunos de estos para alcanzar el equilibrio térmico.

Dean, Breslin y Ross (2014) proponen una actividad experimental que tiene como propósito demostrar cómo el cuerpo mantiene una temperatura interna constante a pesar de los cambios percibidos mediante la sensación térmica, así como generar discusiones sobre mecanismos termorreguladores, como la sudoración y vasodilatación. Es una actividad sencilla que ofrece una demostración de cómo el cuerpo mantiene una temperatura interna constante, a pesar de los cambios en la temperatura de la piel. Los estudiantes deben medir la temperatura corporal central y la sensación térmica antes y después del ejercicio. Al analizar los cambios, como la sudoración y la vasodilatación, se pueden analizar los procesos de termorregulación

Otras actividades tienen objetivos de aprendizaje específicos para la educación superior, como examinar valores de tolerancia térmica o puntos de referencia para definir el gradiente térmico y estimar la temperatura corporal en lagartos (KINGSBURY 1999), analizar la relación entre metabolismo y temperatura, consumo de oxígeno y tasa metabólica en endotermos y ectotermos (HIEBERT \& NOVERAL 2007) o describir la función de los nervios simpáticos vasoconstrictores en la regulación del flujo sanguíneo de la piel (TANSEY, ROE \& JOHNSON, 2014). A partir de ello pueden discutir sobre organismos termoconformes (aquellos organismos cuya temperatura corporal generalmente es semejante a la del ambiente ya que dependen únicamente de dicha temperatura para mantener la propia) y las diferencias entre diferentes grupos de animales termorreguladores (CLOPTON, 2007).

Estas actividades también describen de forma detallada qué adaptaciones didácticas pueden hacerse según el propósito, y aunque que si bien no se ajustan por su profundidad a la educación secundaria, generan ideas metodológicas y reafirman la necesidad de usar actividades centradas en la experimentación, manejo y organización de datos, así como abordajes que permitan establecer conexiones entre conceptos.

Un trabajo interdisciplinar ofrece elementos para que los estudiantes establezcan estas conexiones a partir de las interacciones y el diálogo desde diversas disciplinas. Mulligan et al. (2011), mediante una práctica de laboratorio con estudiantes de un curso de laboratorio de química analítica y un curso de fisiología 
del ejercicio, encontraron resultados favorables en relación con la percepción de los estudiantes sobre este tipo de actividades. La práctica les permitió contextualizar y comprender conceptos del plan de estudios de cada carrera desde otra perspectiva tras examinar el papel de los electrolitos y su importancia en las estrategias de reposición de líquidos para atletas. También les posibilitó analizar las variables asociadas con la termorregulación durante el ejercicio.

Al respecto, Reinartz-Estrada (2012) afirma que incluir referencias de otras ciencias en la explicación de un concepto fisiológico como termorregulación torna más probable que los estudiantes lo identifiquen como un fenómeno que puede ser tratado desde varias disciplinas. Mediante un enfoque problémico en la enseñanza de la fisiología animal con estudiantes de zootecnia, el autor alcanzó resultados positivos en la construcción, por ellos, de conceptos sobre termorregulación. Los estudiantes reconocieron, por ejemplo, que la termorregulación contribuye con la homeóstasis. Contrario a lo anterior, un estudio con profesores en formación inicial desarrollado por la autora de la presente investigación reflejó en ellos dificultades para elaborar explicaciones que trasciendan el sentido común e involucren abordajes integrales de la termorregulación (DAZA-PÉREZ \& PÉREZ-MIRANDA, 2011).

De manera general, los trabajos revisados evidencian la necesidad de fortalecer la investigación a nivel de básica secundaria sobre propuestas de enseñanza que favorezcan la comprensión del concepto de termorregulación y su relación con la homeóstasis, así como la necesidad de abordajes integrales, la inclusión de situaciones problema y la experimentación como elementos básicos para superar concepciones de sentido común, así como para entender la interrelación entre la temperatura y diferentes actividades.

Otros trabajos que han estudiado termorregulación desde la homeóstasis la abordan como un concepto que facilitaría la comprensión de esta última, reconociéndola como una propiedad de los sistemas biológicos. Modell et al. (2015) consideran que los problemas en el aprendizaje sobre termorregulación pueden deberse a la complejidad de los mecanismos de regulación homeostática, a que hay aspectos del fenómeno que son contra intuitivos, que el lenguaje o terminología utilizada en el contexto escolar para describir el fenómeno o concepto es inconsistente y a que la comprensión del fenómeno por la fisiología es aún incierta o incompleta. Así, proponen adoptar cinco estrategias que proporcionarán a los estudiantes un marco coherente para la construcción de sus propios modelos mentales de los mecanismos homeostáticos específicos y ayudarán a reconocer las similitudes funcionales entre los diferentes sistemas de regulación homeostática a nivel del organismo. Estas cinco estrategias son: emplear un conjunto estándar de términos relacionados con el fenómeno, capacitar a los profesores para su enseñanza, definir y usar un modelo estándar, usar de forma cuidadosa analogías ilustrativas y capacitar a los profesores para el uso de dichas analogías. Además, restringir el uso del término 'regulación homeostática' para referirse preferiblemente a los mecanismos relacionados con el mantenimiento de la consistencia del medio interno. 


\section{Documentos curriculares y comentarios de profesores.}

En Colombia, en los estándares básicos de competencia en Ciencias Naturales para la educación básica propuestos por el Ministerio de Educación Nacional (MEN), que constituyen el documento de referencia curricular desde 2004, el concepto de termorregulación no es explícito. Sin embargo, por la organización secuencial que atiende a grupos de grados y la estructura horizontal que permite articular procesos de enseñanza y aprendizaje con los que anteceden o siguen, es posible asociarlo a diversos estándares propuestos para los diferentes grados.

Así, en los grados sexto y séptimo puede ser abordado cuando se trabajen en el aula los siguientes procesos de pensamiento y acciones concretas citadas por los estándares básicos de competencia: comparación de mecanismos de obtención de energía en los seres vivos, identificación de condiciones de cambio y de equilibrio en los seres vivos y en los ecosistemas, y explicación de las condiciones de cambio y conservación en diversos sistemas teniendo en cuenta transferencia y transporte de energía y su interacción con la materia. En los grados octavo y noveno: clasificación de organismos en grupos taxonómicos de acuerdo con sus características celulares y comparación de sistemas de órganos de diferentes grupos taxonómicos. En los grados décimo y undécimo: explicación y comparación de algunas adaptaciones de seres vivos en ecosistemas del mundo y de Colombia, y utilización de modelos biológicos, físicos y químicos para explicar la transformación y conservación de la energía.

En las temáticas del componente biológico o entorno vivo evaluadas en las Pruebas Saber (ICFES 2013 y 2014), la homeóstasis constituye una temática central. La evaluación de este componente se centraliza en el organismo para entender sus procesos internos y sus relaciones con los medios físico y biótico. Considera, además, las relaciones ecológicas, la evolución y transformación de la vida en el planeta y la conservación de la energía.

No obstante, el MEN, en las nuevas propuestas curriculares, recientemente publicó los Derechos Básicos de Aprendizaje (DBA) para Ciencias Naturales y en ese documento propone que a nivel del grado octavo de la educación básica los estudiantes deben:

Analizar relaciones entre sistemas de órganos (excretor, inmune, nervioso, endocrino, óseo y muscular) con los procesos de regulación de las funciones en los seres vivos. (MEN 2016, p. 28).

Ello implica también que se puedan observar en los estudiantes las siguientes evidencias de aprendizaje:

Relacionen los fenómenos homeostáticos de los organismos con el funcionamiento de órganos y sistemas. Interpreten modelos de equilibrio existente entre algunos de los sistemas (excretor, inmune, nervioso, endocrino, óseo y muscular). Relacionen el papel biológico de las hormonas y las neuronas ${ }^{2}$ en la regulación y coordinación del funcionamiento de los sistemas del organismo y el mantenimiento de la homeostasis, dando ejemplos para funciones como la reproducción sexual, la digestión de los alimentos, la regulación de la presión sanguínea y la respuesta de 'lucha o huida'. Expliquen, a través de ejemplos, los efectos de hábitos no saludables en el funcionamiento adecuado de los sistemas excretor, 
nervioso, inmune, endocrino, óseo y muscular" (MEN 2016, p. 28).

Para el MEN estos aprendizajes pueden ser evidentes en una situación como la siguiente (MEN 2016, p. 29):

Explica a partir de la imagen (Figura 1) la interacción entre sistemas del cuerpo humano, al reconocer como el sistema endocrino interviene en el equilibrio homeostático del aparato excretor; predice además, lo que puede ocurrir con los músculos si se afecta el sistema circulatorio y cómo actúa el sistema inmune en el mantenimiento homeostático del cuerpo.

Figura 1. Representación de interacción entre sistemas para alcanzar la homeóstasis. Tomada de los DBA para Ciencias Naturales (MEN 2016, p. 29)

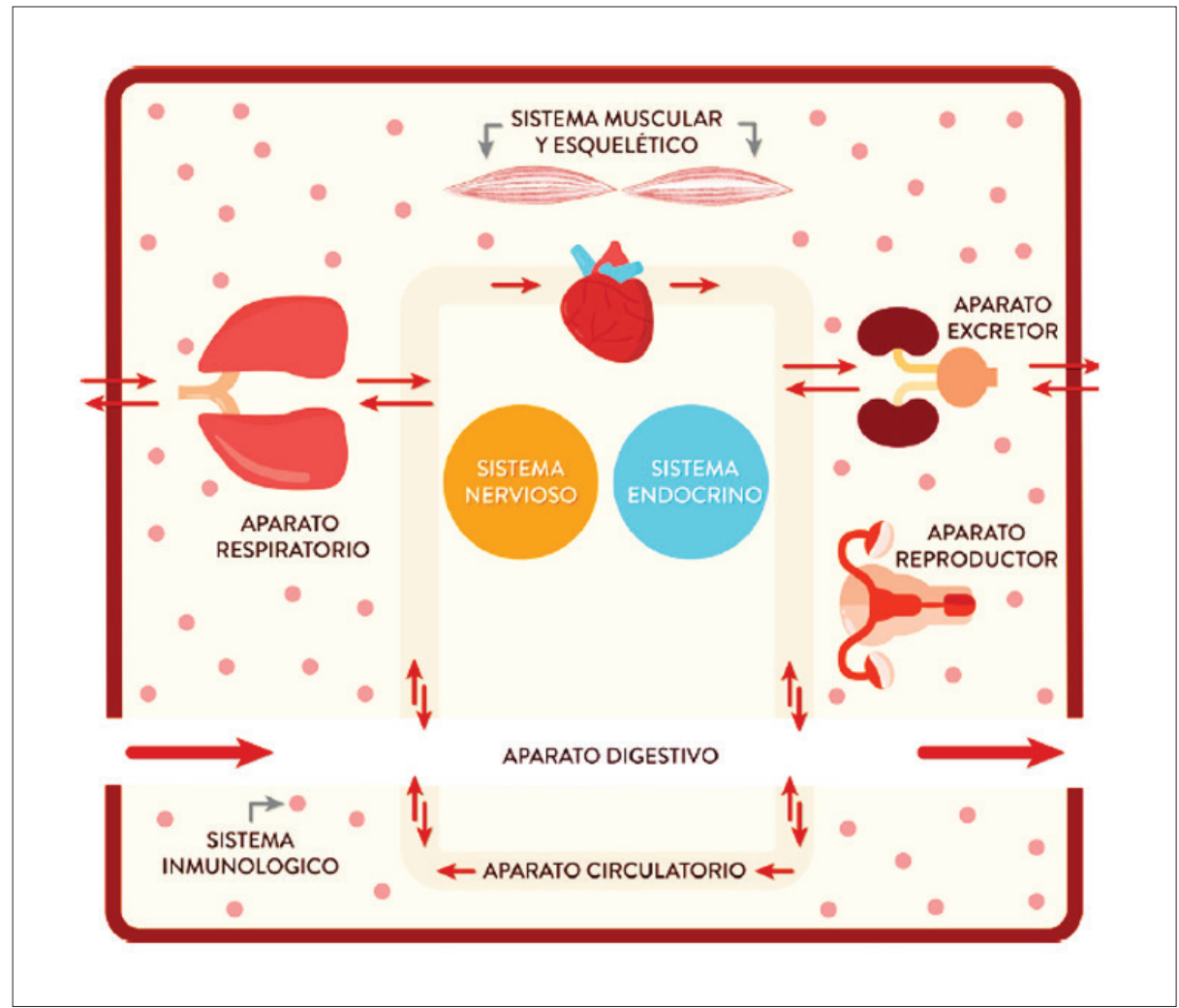

Siendo esta propuesta un documento oficial que indica aquellos conocimientos básicos que un estudiante debe saber en cada grado, es posible concluir que la termorregulación en animales es una temática que provee un contexto pertinente para atender las metas de formación en ciencias que, en este caso, son coherentes con una compresión integral de los fenómenos biológicos que contribuya con la superación de visiones aisladas de los fenómenos biológicos. 
En los 16 profesores encuestados se identificó que cuatro de ellos (25\%) no hacen objeto de enseñanza el concepto. Los demás profesores indican que citan ejemplos de animales ectotermos y endotermos en los diferentes grados según el tema que traten: respiración, circulación, adaptaciones, evolución, homeóstasis, sistema nervioso o flujo de energía en los ecosistemas. Esta falta de unicidad frente a su tratamiento en el aula por parte de los profesores parece tener relación con lo plasmado en los estándares, puesto que allí el concepto no es citado directamente en un grado específico. Asociar el concepto con diversas temáticas es un indicador positivo en la medida de sus conexiones con otros conceptos. Sin embargo, la simple mención del mismo en diferentes temáticas sin una conexión consistente es una limitación que favorece visiones fragmentadas de los fenómenos biológicos involucrados.

Los profesores también indicaron los libros didácticos que utilizan en su práctica. En los libros más utilizados (ÁRBELAEZ et. al., 2004; CARRILLO et. al., 2010; RODRIGUEZ et. al., 2013; LÓPEZ et. al., 2013) verificamos que termorregulación en animales es tratada solamente de modo superficial, en conexión con contenidos sobre evolución y funciones vitales (Tabla 1). También, algunas afirmaciones fortalecen explicaciones de fenómenos y procesos en los animales de forma aislada, haciendo referencia a un solo órgano o sistema.

La aseveración de que la endotermia es una ventaja en comparación con la ectotermia, por ejemplo, merece un tratamiento cuidadoso, que integre fisiología y evolución, puesto que la morfología y fisiología de ectotermos, como reptiles, es una adaptación para funcionar con bajas tasas de energía. Constituye un raciocinio evolutivamente incorrecto afirmar que ella es una desventaja en relación con la endotermia como si la evolución fuese progresiva, con los endotermos situados arriba de los ectotermos en una especie de escala de complejidad.

Otros autores también han identificado limitaciones de los libros de texto que inciden en el aula, debilitando el poder del concepto como una temática central que permite abordajes integrales. Para Tansey y Johnson (2015), en el nivel universitario el concepto de termorregulación a menudo es tratado con superficialidad en la mayoría de los cursos básicos de fisiología para medicina, biología o carreras afines, a pesar de que la investigación en este campo ha progresado a lo largo de las últimas décadas. Se ha profundizado particularmente el conocimiento de los circuitos centrales implicados en el control de termorregulación y de los mecanismos de transducción sensorial periférica de temperatura, pero estos avances no son citados en los libros de texto usados en la enseñanza superior.

Dificultades similares asociadas al concepto de homeóstasis han sido encontradas también en textos de fisiología. Para Modell et al. (2015), las explicaciones presentadas en cerca de 11 textos universitarios no citan de forma coherente términos relacionados con la regulación homeostática. Además, varios textos no utilizan representaciones visuales coherentes con el concepto y la explicación del concepto a menudo entra en conflicto con el conocimiento actual de los mecanismos reguladores homeostáticos. 
Tabla 1. Aspectos sobre termorregulación citados en los libros de texto analizados.

\begin{tabular}{|c|c|c|}
\hline Libro de texto & $\begin{array}{c}\text { Temáticas } \\
\text { asociadas con } \\
\text { termorregulación }\end{array}$ & Observaciones \\
\hline \multirow{2}{*}{$\begin{array}{l}\text { Contextos } \\
\text { Naturales } 9 \\
\text { (ÁRBELAEZ et. } \\
\text { al., 2004). }\end{array}$} & \multirow{2}{*}{$\begin{array}{l}\text { Unidad: } \\
\text { “Caminos } \\
\text { evolutivos y } \\
\text { ecosistemas". } \\
\text { Apartado: } \\
\text { Temperatura: } \\
\text { Los organismos } \\
\text { endotermos. }\end{array}$} & $\begin{array}{l}\text { Describe la ectotermia e indica que dos } \\
\text { grupos de animales lograron desarrollar } \\
\text { una forma de mantener la temperatura } \\
\text { corporal constante e independiente } \\
\text { de la temperatura ambiental. Según } \\
\text { el texto las aves y los mamíferos } \\
\text { son endotermos... " (endo: dentro; } \\
\text { termo: temperatura), ya que tienen } \\
\text { capas de pelos y plumas que aíslan su } \\
\text { temperatura corporal. Pueden tener un } \\
\text { metabolismo alto sin mucha pérdida de } \\
\text { energía debido al escape de calor hacia } \\
\text { el ambiente"... (p. 115). }\end{array}$ \\
\hline & & $\begin{array}{l}\text { Afirma que ser endotermo tiene muchas } \\
\text { ventajas y cita tres de estas. 1. La } \\
\text { actividad no está limitada a épocas del } \\
\text { año u horas del día ya que se evita la } \\
\text { pérdida de energía continua en forma } \\
\text { de calor. } 2 \text {. No corren riesgos derivados } \\
\text { de situaciones prolongadas de letargo } \\
\text { o hibernación. Como el metabolismo } \\
\text { es elevado, pueden hacer esfuerzos } \\
\text { constantes como correr o volar. } 3 \text {. Pueden } \\
\text { ocupar hábitats de bajas temperaturas } \\
\text { permanentemente (p. 115). }\end{array}$ \\
\hline $\begin{array}{l}\text { Hipertexto } 9 \\
\text { (CARRILLO et. } \\
\text { al., 2010). }\end{array}$ & $\begin{array}{l}\text { Unidad: } \\
\text { “los seres vivos } \\
\text { evolucionan”. } \\
\text { Sección: } \\
\text { Reptiles. Control } \\
\text { de la } \\
\text { temperatura. }\end{array}$ & $\begin{array}{l}\text { Se refiere a la ectotermia como } \\
\text { característica que dificultó la colonización } \\
\text { de hábitats muy fríos...."La endotermia } \\
\text { permitió a los animales mantenerse } \\
\text { activos durante más tiempo, lo que les } \\
\text { dio ventajas selectivas que con el paso } \\
\text { del tiempo favorecieron la evolución de } \\
\text { las aves y mamíferos"... (p. 101) }\end{array}$ \\
\hline
\end{tabular}




\begin{tabular}{|c|c|c|}
\hline \multirow{4}{*}{$\begin{array}{l}\text { Los caminos } \\
\text { del saber } 6 \\
\text { (RODRIGUEZ } \\
\text { et. al., 2013). }\end{array}$} & \multirow{4}{*}{$\begin{array}{l}\text { Unidad: } \\
\text { “Funciones } \\
\text { vitales". } \\
\text { Apartado: } \\
\text { autorregulación- } \\
\text { equilíbrio interno } \\
\text { u homeostasis. }\end{array}$} & $\begin{array}{l}\text { En una tabla indica que la temperatura } \\
\text { de los reptiles, peces y anfibios fluctúa } \\
\text { de acuerdo con el medio. }\end{array}$ \\
\hline & & $\begin{array}{l}\text { Cuando se refiere a organismos } \\
\text { pluricelulares tratando del medio } \\
\text { interno, cita homeostasis o equilibrio del } \\
\text { medio interno, como una capacidad de la } \\
\text { pluricelularidad. Esto incluye regulación } \\
\text { de la temperatura. }\end{array}$ \\
\hline & & $\begin{array}{l}\text { Subtitulo control de la temperatura } \\
\text { interna: el control de la temperatura } \\
\text { interna constituye otro atributo } \\
\text { evolutivo importante propio de los } \\
\text { endotermos, que les permite mantener } \\
\text { una temperatura interna constante } \\
\text { independiente del ambiente, gracias al } \\
\text { calor generado en el metabolismo (p. 44). }\end{array}$ \\
\hline & & $\begin{array}{l}\text { Presenta imagen con nota aclaratoria } \\
\text { que dice: los peces, anfibios y reptiles } \\
\text { son organismos ectotermos que } \\
\text { obtienen el calor del ambiente y no de } \\
\text { sus procesos metabólicos (p.44). }\end{array}$ \\
\hline \multirow[b]{2}{*}{$\begin{array}{l}\text { Los caminos } \\
\text { del saber } 8 \\
\text { (López et. al., } \\
\text { 2013). }\end{array}$} & \multirow[b]{2}{*}{$\begin{array}{l}\text { No cita } \\
\text { directamente } \\
\text { el tema. }\end{array}$} & $\begin{array}{l}\text { Control y regulación: Sistema nervioso } \\
\text { central en el ser humano. En tabla sobre } \\
\text { función del hipotálamo, apunta que, entre } \\
\text { otras funciones, participa en la regulación } \\
\text { de la temperatura corporal. (p. 130). }\end{array}$ \\
\hline & & $\begin{array}{l}\text { Al discutir adaptaciones fisiológicas } \\
\text { afirma: "son modificaciones metabólicas } \\
\text { que permiten a los individuos } \\
\text { sobrevivir y que están relacionadas } \\
\text { con procesos de transformación de } \\
\text { energía y reacciones químicas entre } \\
\text { otras. Por ejemplo, existen organismos } \\
\text { euritermos, que toleran un amplio } \\
\text { rango de temperaturas, y estenotermos, } \\
\text { que sólo pueden vivir en rangos muy } \\
\text { estrechos" (p 200). }\end{array}$ \\
\hline
\end{tabular}

Fuente: elaborada por los autores (2018). 
La literatura consultada, los libros de texto y las respuestas de los profesores al cuestionario evidencian la necesidad de un tratamiento integrado y explícito sobre termorregulación como mecanismo homeostático en educación básica secundaria. Se requiere además que se supere la naturaleza puramente descriptiva de los fenómenos fisiológicos, para que el estudiante logre adentrarse en relaciones causales que son necesarias para dar sentido a los mecanismos. Los estudiantes deben analizar el fenómeno desde varias perspectivas, así como acudir a conceptos de diferentes disciplinas para elegir un marco de referencia apropiado desde el cual analizar aspectos del mismo (MODELL 2007).

En coherencia con lo expuesto, a continuación se detalla sobre un abordaje de termorregulación que sigue planteamientos filosóficos sobre mecanismos biológicos. Proponemos hacer objeto de estudio la termorregulación desde la perspectiva de mecanismo homeostático como una alternativa para organizar sistemas integrados de pensamiento, que proporcionen a los estudiantes la posibilidad de integrar los diferentes componentes y procesos en los sistemas vivos, tener una visión más completa de los mismos, su complejidad e interacciones con el medio externo (ASSRAF et al. 2013).

\section{TERMORREGULACIÓN COMO MECANISMO DE REGULACIÓN HOMEOSTÁTICA}

En la filosofía de la biología, hoy la naturaleza de la explicación biológica es comprendida de manera más pluralista, en comparación con el énfasis en el pasado sobre explicaciones deductivas basadas en leyes similares a las leyes físicas (de acuerdo con el llamado modelo deductivo-nomológico) (como discutido, e.g., por BRIGANDT, 2013). Entre las diversas maneras de explicar en biología, hay una que ha recibido especial atención en las últimas tres décadas, las explicaciones por medio de la proposición de modelos de mecanismos (e.g., MACHAMER, DARDEN \& CRAVER 2000; BECHTEL \& RICHARDSON 2010; CRAVER \& DARDEN 2013). Estas explicaciones tienen un papel central en el estudio de los fenómenos vivos, aunque que no sean, de modo alguno, la única manera de explicar en Biología. Sobre mecanismos se han propuesto varias definiciones (e.g., MACHAMER, DARDEN \& CRAVER 2000; GLENNAN 2002; BECHTEL 2006) cuyos puntos de acuerdo fundamentan la propuesta que aquí se plantea. Se reconoce entonces que un mecanismo consta de varios componentes o partes que están organizadas y cada parte o componente desempeña una función e interactúa con las demás de forma coordinada para producir un fenómeno determinado. Sin embargo, siguiendo a Machamer, Darden y Craver (2000, p.3) la caracterización que se ajusta a las pretensiones didácticas del presente trabajo es la de mecanismo como:

Un conjunto de entidades y actividades organizadas de tal manera que producen cambios regulares desde las condiciones de inicio o de establecimiento hasta las condiciones de finalización o de terminación [...]

Así, primero identificamos el aspecto fenomenal del mecanismo de interés, es decir, el fenómeno que es producido por el mecanismo, que en este caso es: mantener la temperatura interna en rangos de tolerancia y desempeño que 
son específicos para las diferentes especies, esto es, mantener la homeóstasis en la temperatura interna. Seguidamente se identificamos las partes o los componentes del mecanismo, las actividades que realizan cada componente, cómo se organizan y conectan las diferentes actividades (Tabla 2).

En la Tabla 2, presentamos los componentes, las actividades que realizan y las relaciones que existen entre cada uno de ellos, lo cual fue básico para estructurar el modelo didáctico que representa y explica los elementos centrales que se consideran básicos en la comprensión del mecanismo de la termorregulación.

Los componentes del mecanismo son básicamente los de un sistema de regulación homeostático (termorreceptores, punto de ajuste y efectores). La actividad metabólica, específicamente el catabolismo, es resaltado como otro componente cuya actividad de transformación de la energía determina diferencias en la termorregulación de los animales y está regulada por los sistemas endocrino y nervioso. El calor metabólico, como se le llama con frecuencia, es otra vía por la cual un animal puede ganar energía térmica.

A nivel celular la mayoría de los ectotermos regulan la capacidad metabólica de sus tejidos en respuesta a la variación térmica a largo plazo (días a semanas) (SEEBACHER, 2005). En ellos, contrario a lo que ocurre con los endotermos, donde el metabolismo celular también genera la energía térmica que determina la temperatura corporal [termogénesis adaptativa (MORRISON et al., $\left.2008^{3}\right)$ ], la interacción entre la temperatura corporal y el metabolismo se invierte. En su mayoría, no desarrollan termogénesis adaptativa y aclimatan el metabolismo celular para compensar la variación de temperatura ambiental. Por ejemplo, la tasa metabólica de los lagartos es baja a temperaturas frías y alta a temperaturas cálidas. 
Tabla 2. Organización de componentes y actividades de un mecanismo para termorregulación en vertebrados terrestres basada la propuesta general de mecanismo de Darden (2008), adaptada de van Mil, Boerwinkel y Waarlo (2011)

\begin{tabular}{|c|c|c|c|c|c|c|}
\hline \multicolumn{3}{|c|}{ Componentes } & \multirow{3}{*}{ Localización } & \multirow{3}{*}{ Función } & \multirow{3}{*}{$\begin{array}{c}\text { Conectividad } \\
\text { ¿Qué } \\
\text { entidades } \\
\text { y/o } \\
\text { actividades } \\
\text { están } \\
\text { conectadas } \\
\text { en el } \\
\text { mecanismo? }\end{array}$} & \multirow{3}{*}{ Orden } \\
\hline \multicolumn{2}{|c|}{ Entes } & \multirow{2}{*}{ Actividades } & & & & \\
\hline $\begin{array}{c}\text { Papel } \\
\text { Funcional }\end{array}$ & $\begin{array}{c}\text { Papel } \\
\text { Estructural }\end{array}$ & & & & & \\
\hline $\begin{array}{l}\text { Termor- } \\
\text { receptores }\end{array}$ & & & $\begin{array}{l}\text { Piel, } \\
\text { lengua, } \\
\text { córnea, } \\
\text { vejiga, } \\
\text { sistema } \\
\text { nervioso } \\
\text { central }\end{array}$ & $\begin{array}{l}\text { Sensitiva. Mide } \\
\text { el valor de la } \\
\text { temperatura y } \\
\text { envía señalas } \\
\text { al centro de } \\
\text { control para } \\
\text { que detecte } \\
\text { variaciones }\end{array}$ & $\begin{array}{c}\text { Sistema } \\
\text { nervioso } \\
\text { central }\end{array}$ & \multirow{9}{*}{$\begin{array}{l}\text { 1. Detección de } \\
\text { la pérdida de la } \\
\text { homeóstasis de } \\
\text { la temperatura } \\
\text { interna. } \\
\text { 2. Información } \\
\text { procesada en } \\
\text { el Sistema } \\
\text { Nervioso } \\
\text { Central. } \\
\text { 3. Se genera } \\
\text { respuesta: } \\
\text { 4. Aumento o } \\
\text { disminución del } \\
\text { metabolismo. } \\
\text { Respuestas } \\
\text { fisiológicas. } \\
\text { Respuestas } \\
\text { comportamen- } \\
\text { tales }\end{array}$} \\
\hline \multirow{3}{*}{$\begin{array}{l}\text { Centro de } \\
\text { control - Punto } \\
\text { de ajuste }\end{array}$} & $\begin{array}{c}\text { Sistema } \\
\text { nervioso } \\
\text { central - } \\
\text { termostato } \\
\text { hipotalámico }\end{array}$ & & $\begin{array}{l}\text { Columna } \\
\text { vertebral, } \\
\text { cráneo }\end{array}$ & & $\begin{array}{l}\text { Sistema } \\
\text { endocrino, } \\
\text { termor- } \\
\text { receptores, } \\
\text { efectores }\end{array}$ & \\
\hline & $\begin{array}{l}\text { Sistema } \\
\text { endocrino }\end{array}$ & & $\begin{array}{l}\text { Diferentes } \\
\text { glándulas } \\
\text { distribuidas } \\
\text { en los } \\
\text { órganos }\end{array}$ & $\begin{array}{l}\text { actividad } \\
\text { nerviosa, } \\
\text { la actividad } \\
\text { metabólica, y la } \\
\text { producción de } \\
\text { sudor. }\end{array}$ & $\begin{array}{l}\text { Sistema } \\
\text { nervioso, } \\
\text { termor- } \\
\text { receptores, } \\
\text { efectores }\end{array}$ & \\
\hline & & $\begin{array}{c}\text { Actividad } \\
\text { metabólica } \\
\text { - Vías } \\
\text { catabólicas }\end{array}$ & $\begin{array}{l}\text { A nivel } \\
\text { celular }\end{array}$ & $\begin{array}{l}\text { Transforma } \\
\text { energía } \\
\text { química en } \\
\text { térmica y libre }\end{array}$ & $\begin{array}{l}\text { Sistema } \\
\text { endocrino, } \\
\text { sistema } \\
\text { nervioso }\end{array}$ & \\
\hline \multirow[t]{5}{*}{ Efectores } & $\begin{array}{c}\text { Músculos, } \\
\text { vasos } \\
\text { sanguíneos, } \\
\text { piel }\end{array}$ & & $\begin{array}{l}\text { Todo el } \\
\text { cuerpo }\end{array}$ & $\begin{array}{l}\text { Ejecutan } \\
\text { respuestas } \\
\text { fisiológicas } \\
\text { y comporta- } \\
\text { mentales }\end{array}$ & $\begin{array}{l}\text { Sistema } \\
\text { endocrino } \\
\text { y sistema } \\
\text { nervioso }\end{array}$ & \\
\hline & & Convección ${ }^{4}$ & \multirow{4}{*}{ No se aplica } & \multirow{3}{*}{$\begin{array}{l}\text { Gaño o pierda } \\
\text { de energía } \\
\text { en relación } \\
\text { al ambiente } \\
\text { externo }\end{array}$} & \multirow{4}{*}{$\begin{array}{c}\text { Está ligado } \\
\text { con todos los } \\
\text { componentes. } \\
\text { Es una } \\
\text { propiedad } \\
\text { de todos } \\
\text { los cuerpos } \\
\text { el perder o } \\
\text { ganar energía } \\
\text { a través de } \\
\text { estas vías } \\
\text { físicas }\end{array}$} & \\
\hline & & Conducción & & & & \\
\hline & & Radiación & & & & \\
\hline & & Evaporación & & $\begin{array}{l}\text { Cede energía } \\
\text { al ambiente } \\
\text { externo }\end{array}$ & & \\
\hline
\end{tabular}

Fuente: elaborada por los autores (2018). 
Los endotermos, por su parte, tienen tasas metabólicas que son siete a diez veces mayores que la de ectotermos del mismo tamaño. Además, disminuyen la producción de energía térmica en situaciones donde la temperatura externa está en valores muy por encima de los rangos específicos de cada especie o pueden aumentarla en el caso contrario.

Durante el metabolismo celular, cuando moléculas como la glucosa participan de reacciones como reactantes, la energía de los productos de la reacción es menor que la energía originalmente presente en los reactantes y la energía libre resultante de esta reacción exergónica es utilizada para la producción de trabajo o la formación de moléculas envueltas en las trocas energéticas en la célula, como el ATP. Una parte de la energía libre, sin embargo, es liberada en forma de energía térmica. Esta energía no aprovechada es la energía térmica que los endotermos utilizan para mantener su temperatura en niveles relativamente estables y óptimos de manera que exista un equilibrio interno u homeóstasis. Cuando dicho equilibrio es alterado debido a un desbalance de energía ganada, se produce la regulación de la temperatura.

En consecuencia con lo anterior, nuestra propuesta de modelo didáctico sobre termorregulación sigue el modelo simplificado de homeóstasis propuesto por Modell et al. (2015) y se centra en la termorregulación como un mecanismo asociado con el mantenimiento de la homeóstasis (Figura 2).

Figura 2. Representación simplificada de un sistema de regulación homeostático centrado en la regulación de la temperatura

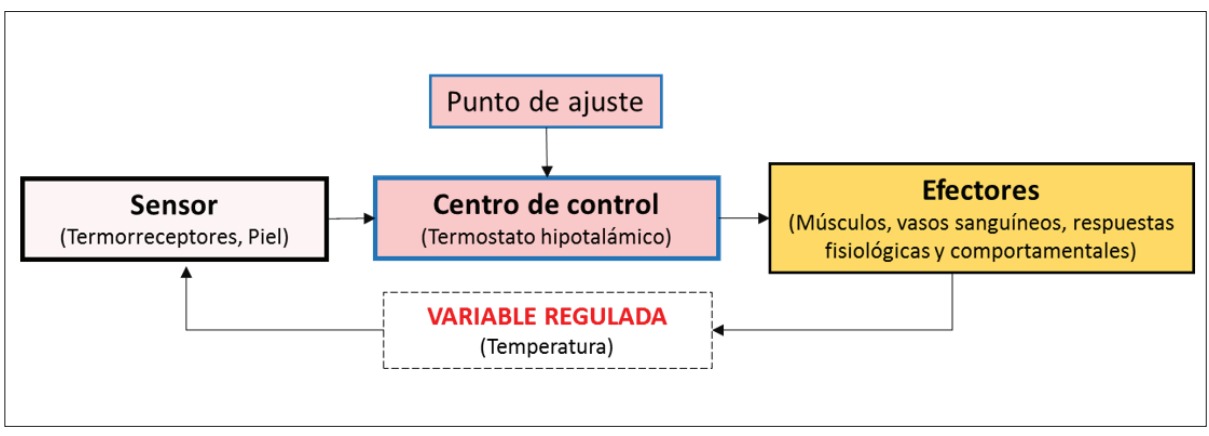

Fuente: elaborada por los autores (2018)

El modelo comprende los siguientes elementos:

Punto de ajuste: el rango de valores (rango de magnitudes) de la variable regulada que el sistema intenta mantener. Se refiere a la meta de regulación del sistema, el valor que el sistema mantiene en niveles relativamente estables y óptimos, en consecuencia de procesos evolutivos anteriores. Esto generalmente no es un valor único, sino un rango de valores.

Centro de control (o integrador): el centro de control consta de un detector de errores y un controlador. Recibe señales (información) de sensores, compara información (valor de variable regulada) con el punto establecido, integra información de todos los sensores y envía señales de salida (envía instrucciones o comandos) para aumentar o disminuir la actividad de los efectores. El centro de control determina e inicia la respuesta fisiológica apropiada a cualquier cambio o perturbación del ambiente interno. 
Efector: Un componente cuya actividad o acción contribuye a determinar el valor de cualquier variable del sistema. En este modelo, los efectores determinan el valor de la variable regulada (detectada).

Variable regulada (variable detectada): Cualquier variable para la cual los sensores están presentes en el sistema y cuyo valor se mantiene dentro de los límites de un sistema de retroalimentación negativa frente a las perturbaciones.

Sensor (Receptor): Un “dispositivo" que mide la magnitud de alguna variable al generar una señal de salida (neuronal u hormonal) que es proporcional a la magnitud del estímulo. Un sensor es un "dispositivo" de medición.

En el modelo didáctico (Figura 3), que también se apoya en los trabajos de Bicego, Barros y Branco (2007); Kuht y Farmery (2014); Pough et al. (2012); y Vitt y Caldwell (2013), la zona sombreada representa los aspectos centrales del mecanismo. El fenómeno producido es el mantenimiento de la temperatura corporal entre rangos de tolerancia o desempeño óptimos y específicos para cada especie o grupo de especies (Cuadro rojo), es decir, asegurar la homeóstasis en términos de temperatura.

El sistema nervioso central responde a un estímulo: la pérdida de la homeóstasis de la temperatura (desequilibrio homeostático), es decir, el hecho de que la temperatura corporal interna está por debajo o supera los rangos de tolerancia específicos para cada especie. Este cambio es detectado a nivel del sistema nervioso central (medula espinal, tronco encefálico, encéfalo) a partir de la información que ha sido generada por los termorreceptores.

Figura 3. Modelo didáctico del mecanismo de termorregulación en vertebrados terrestres

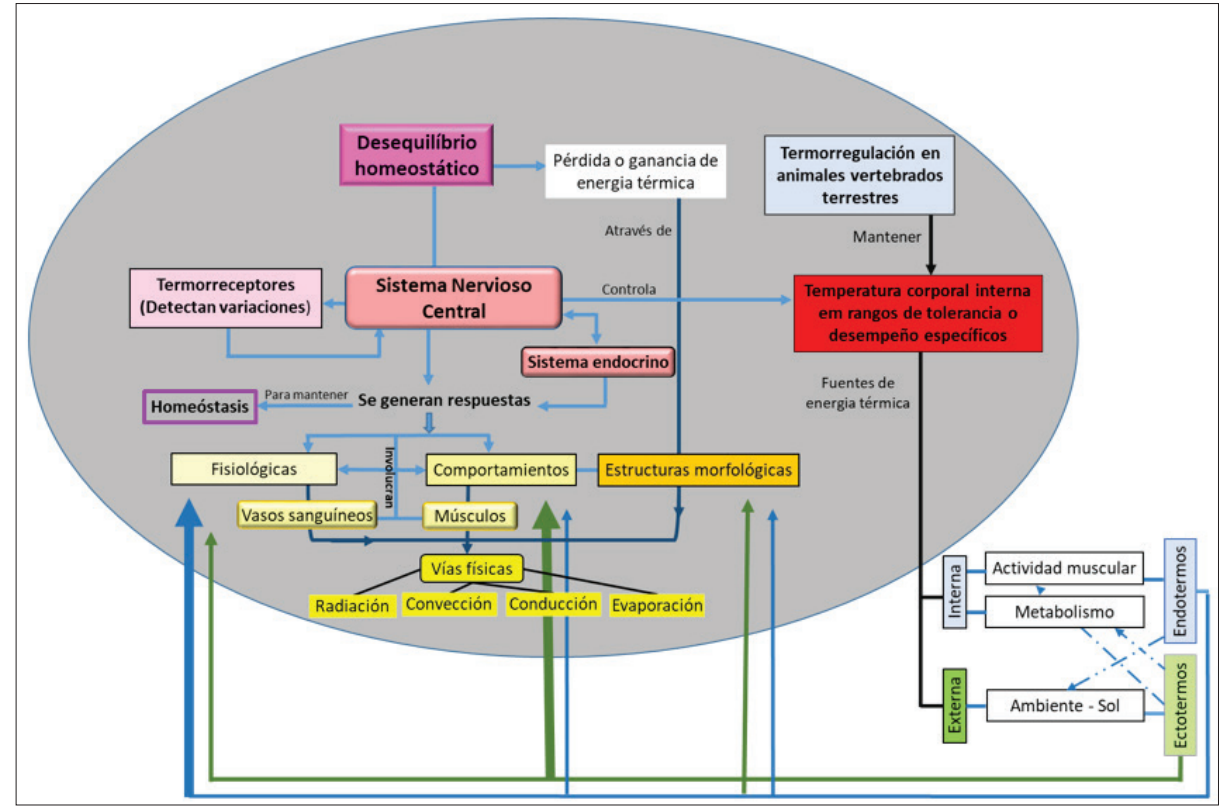

Fuente: Elaborada por los autores (2018). 
En el sistema nervioso central se procesa la información y generan respuestas básicamente fisiológicas, que se manifiestan en comportamientos del animal o ajustes fisiológicos en sí, que controlan la temperatura. El sistema endocrino no sólo complementa la función del sistema nervioso. Existe un control mutuo de sus acciones. En este caso el sistema endocrino actúa a nivel del metabolismo, influenciando la sudoración, la vasoconstricción o vasodilatación.

Las respuestas citadas en el diagrama son vías de pérdida y ganancia de energía térmica ligadas entre sí porque una no es independiente de la otra y no es la única respuesta. Es decir, un animal puede moverse a un lugar soleado o modificar el color de su piel para ganar energía térmica por radiación, o ampliar su superficie corporal para ganar o perder energía más rápidamente por conducción o radiación. En caso de estas vías físicas, a diferencia de otros sistemas, la transferencia no logra el equilibrio térmico entre los dos sistemas: la temperatura interna de los animales se mantiene entre rangos específicos.

La actividad física permite generar energía térmica por actividad muscular: correr, caminar o quedarse quieto son comportamientos ligados a la producción de energía térmica.

Las respuestas de tipo fisiológico y comportamental están conectadas por una doble flecha en la figura 3 porque se asume que el comportamiento de los animales es producto en parte de diferentes procesos fisiológicos y cada comportamiento también genera las condiciones para que se lleven a cabo procesos fisiológicos.

En el modelo, fuera de la zona sombreada también se indican diferencias entre los animales en función de la principal fuente de energía térmica: interna - endotermos; externa - ectotermos. Las flechas punteadas indican fuentes de energía secundarias para ambos.

Dada la dependencia térmica del ambiente externo en los ectotermos, su principal mecanismo de regulación de la temperatura es comportamental (flecha verde resaltada) mientras que en los endotermos es fisiológica (flecha azul resaltada). Las fechas azul y verde no resaltadas también señalan otros mecanismos de pérdida y ganancia de energía por endotermos y ectotermos.

\section{CONSIDERACIONES FINALES}

Las limitaciones en cuanto al tratamiento didáctico que hacen los libros y los profesores ponen de manifiesto la importancia de un abordaje explícito en conexión con la homeóstasis. Se trata de una de las propiedades de central importancia para los organismos vivos, es decir, cómo los organismos vivos alcanzan una estabilidad interna a pesar de los cambios en el ambiente externo, en este caso, una estabilidad de la temperatura entre rangos específicos.

Termorregulación constituye un concepto central en la enseñanza de la biología no solamente por constituir un mecanismo asociado con la homeóstasis, sino también por su relación con conceptos definidos en el currículo de la educación básica, como se ejemplifica en este artículo en Colombia y, sin embargo, se puede ver en otros países como fundamentales en el aprendizaje de las ciencias naturales: por ejemplo, adaptación, evolución, mecanismos de intercambio de energía, 
calor, temperatura etc. Por ello, su enseñanza ha de ser explícita e integrada. Es importante superar tratamientos superficiales que se limiten a la simple mención de términos técnicos (e.g., endotermos, ectotermos).

El modelo propuesto pretende abordar la termorregulación en los animales siguiendo un enfoque que favorezca la comprensión de la misma como mecanismo homeostático. El puede ser usado como recurso de aprendizaje a nivel de básica secundaria, principalmente en el grado noveno en el caso de Colombia, de tal forma que se consigan integrar conceptos de la física, biología y química definidos como básicos para este grado, como: adaptaciones, mecanismos de pérdida y ganancia de energía de diferentes sistemas, evolución, energía térmica, calor, temperatura, metabolismo.

Sin embargo, siendo la termorregulación en animales el principal concepto objeto de aprendizaje, el modelo didáctico puede ser empleado en los diferentes grados, tomando como punto de partida cualquiera de las siguientes situaciones de enseñanza y aprendizaje asociadas y en diferentes momentos de una sesión de aprendizaje: para introducir, desarrollar, o concluir. Por ejemplo:

1. Discusión de procesos evolutivos. La termorregulación en los diferentes animales tiene una historia evolutiva que implica discusión en el aula de los procesos selectivos que han actuado en relación a este mecanismo, así como las consecuencias de determinada condición (endotermia, ectotermia etc.). Al respecto Eliosa y Silva (2011) consideran que, aunque las aves y los mamíferos tienen distinto origen filogenético, la endotermia asociada con estos grupos tiene los mismos principios fisiológicos, por lo que constituye un carácter convergente (homoplásico). Esto lleva a considerar que la endotermia evolucionó a partir de un mecanismo similar en los dos grupos, como una respuesta a presiones selectivas. En ese sentido, es posible abordar y discutir diversas hipótesis propuestas para explicar evolución de la endotermia por presiones selectivas, como: a) la expansión térmica del nicho; b) la capacidad de mantener un ejercicio vigoroso; y c) una consecuencia del desarrollo de cuidado parental.

2. Abordar adaptaciones en conexión con los procesos evolutivos, dado que el concepto es utilizado frecuentemente con un significado funcional y no evolutivo.

3. Tratamiento de cuestiones termodinámicas, principalmente lo relacionado con los conceptos de energía térmica, temperatura y fenómenos físicos de pérdida o ganancia de energía en sistemas abiertos.

4. Análisis de las respuestas metabólicas, específicamente lo concerniente a vías catabólicas, así como de las causas y consecuencias de tales respuestas en diferentes organismos vivos. 
5. Ejemplificación de mecanismos biológicos, juntamente con diagramas de representación de los mismos.

Sumado a lo anterior y teniendo en cuenta que es un concepto fisiológico, en su comprensión están implicados conceptos explicados desde otras disciplinas, como la física y la química (ya se han ejemplificado algunos) que al ser explorados y citados generan conexiones entre fenómenos y disciplinas.

Abordar termorregulación como mecanismo fisiológico, que contribuye con la homeóstasis, implica la puesta en juego de diversas habilidades, como el identificar componentes, acciones, relaciones; organizar, categorizar y sobre todo establecer relaciones e interrelaciones, lo cual es básico en el desarrollo de visiones integrales, no aisladas de los fenómenos biológicos. Se trata también de comprender termorregulación como un mecanismo que hace parte de un fenómeno global y comprender los submecanismos responsables de regular la temperatura.

\section{AGRADECIMIENTOS}

O presente trabalho foi realizado com apoio da Coordenação de Aperfeiçoamento de Pessoal de Nível Superior - Brasil (CAPES) - Código de Financiamento 001.

Los autores agradecen los especialistas en fisiología: Dra. Nusa de Almeira Silveira (UFG) y Dr. Carlos Arturo Navas Lannini (USP) por su apoyo en la revisión del artículo y validación del diagrama sobre termorregulación. También a los profesores de secundaria que resolvieron el cuestionario.

Erika P. Daza-Pérez, agradece a la OEA y el grupo COIMBRA por la beca de formación doctoral, programa de formación que permitió el desarrollo del artículo.

Charbel N. El-Hani agradece ao CNPq por Bolsa de Produtividade em Pesquisa (Proc. n. 303011/2017-3) e pelo apoio ao Instituto Nacional de Ciência \& Tecnologia em Estudos Interdisciplinares e Transdisciplinares em Ecologia e Evolução (INCT IN-TREE) (Proc. n. 465767/2014-1). Pelo apoio ao INCT INTREE, agradece também à CAPES (Proc. n. 23038.000776/2017-54).

\section{REFERENCIAS}

ÁRBELAEZ, R. et al. Contextos Naturales 9. Colombia: Santillana, 2004.

ASSARAF, O.; DODICK J.; TRIPTO, J. High school students' understanding of the human body system. Res Sci Educ, 43, 33-56, 2013.

BECHTEL, W. Discovering cell mechanisms: The creation of modern cell biology. Cambridge: Cambridge University Press, 2006.

BECHTEL, W.; RICHARDSON, R. C. Discovering complexity: Decomposition and localization as strategies in scientific research. Cambridge: MA. MIT Press, 1993. EE.UU: Princeton University Press, 2010. 
BICEGO, K.; BARROS, R.; BRANCO, L. Physiology of temperature regulation: Comparative aspects. Comparative Biochemistry and Physiology, Part A. 147, 616-639, 2007.

BRIGANDT, I Explanation in Biology: Reduction, Pluralism, and Explanatory Aims. Science \& Education, 22, 69-91, 2013.

BUDDINGH, J. Students' Personal Knowledge of Regulation and Homeostasis: Pioneering in Biology Classrooms. In: The proceedings of the third international seminar on misconceptions and educational strategies in science and mathematics, misconceptions, Trust: Ithaca, NY, 1993.

CARRILLO, A.C. et al. Hipertexto 9. Colombia: Santillana, 2010.

ÇIMER, A. What makes biology learning difficult and effective: Students' views? Educational Research and Reviews, 7, 61-71, 2012.

CLOPTON, J. R. Temperature: Humans Regulating, Ants Conforming. The American Biology Teacher, 69, 5, 59-63, 2009.

CRAVER, C. F.; DARDEN, L. In Search of Mechanisms: Discoveries across the Life Sciences. Chicago, IL: University of Chicago Press, 2013.

DARDEN, L. Thinking again about biological mechanisms. Philos Sci, 75, 958-969, 2008.

DAZA-PÉREZ, E.; PÉREZ-MIRANDA, R. Termorregulación de lagartos en la formación de profesores de ciencias naturales y educación ambiental. Ciência \& Educação (Bauru), 17, 3, 663 678, 2011.

ELIOSA, H.; SILVA A. El origen de la endotermia en los vertebrados. Ciencias, 102, abril-junio, 28-36, 2011.

GLENNAN, S. Rethinking mechanistic explanation. Philosophy of Science, 69(Suppl.), S342S353, 2002.

GROTZER, T. A. Learning to understand the forms of causality implicit in scientifically accepted explanations. Studies in Science Education, 39, 1, 1-74, 2003.

HIEBERT, S.; NOVERAL, J. Are chicken embryos endotherms or ectotherms? A laboratory exercise integrating concepts in thermoregulation and metabolism. Advances in Physiology Education, 31, 1, 97-109, 2007.

JUNGWIRTH, E.; DREYFUS, A. After this, therefore because of this: One-Way of jumping to conclusions. Journal of Biological Education, 26, 139-142, 1992.

KINGSBURY, B. A. An Experimental Design for Examining Thermoregulatory Set Points in Ectothermic Animals. The American Biology Teacher, 61, 6, 1999.

KUHT, J.; FARMERY, A. Body temperature and its regulation. Anaesthesia and intensive care medicine, 15, 6, 273-278, 2014.

LÓPEZ, G. et al. Los caminos del saber 8. Colombia: Santillana, 2013.

MACHAMER, P.; DARDEN L.; CRAVER C. Thinking about mechanisms. Philos Sci. 67, 1-25, 2000.

MINISTERIO DE EDUCACIÓN NACIONAL DE COLOMBIA. Derechos básicos de aprendizaje: Ciencias Naturales. 2016. Disponible en: http://aprende.colombiaaprende.edu.co/ sites/default/files/naspublic/DBA_C.Naturales.pdf. Acceso en: 8 jul. 2018. 
MODELL H. I. Helping students make sense of physiological mechanisms: the "view from the inside." Advan. Physiol. Edu. 31,186-192, 2007.

MODELL H. I. How to help students understand physiology? Emphasize general models. Advan Physiol Educ, 23. 101-107, 2000.

MODELL, H.; CLIFF, W.; MICHAEL, J.; MCFARLAND, J.; WENDEROTH, M.; WRIGHT, A. A physiologist's view of homeostasis. Advances in Physiological Education, 39, 259-266, 2015.

MULligAN, G.; TAYLOR, N.; GLEN, M.; TOMLIN, D.; GAUL, C. Cross-disciplinary thermoregulation and sweat analysis laboratory experiences for undergraduate Chemistry and Exercise Science students. Advances in Physiological Education, 35, 206-212, 2011.

NATIONAL RESEARCH COUNCIL [NRC]. A new biology for the 21st century. Washington, DC: National Academic Press, 2009.

POUGH, H.; JANIS, C. M.; HEISER J. B. Vertebrate Life. San Francisco: Benjamin Cummings, 2012.

REINARTZ-ESTRADA, M. Aportes del enfoque problémico en la enseñanza de la fisiología animal y la conceptualización científica. Revista Iberoamericana de Educación, 59, 3, 2-11, 2012.

RODRIGUEZ, L. E. et al. Los caminos del saber 6. Colombia: Santillana, 2013.

SVOBODA, J.; PASSMORE, C. The Strategies of Modeling in Biology Education. Science \& Education, 22, 119-142, 2013.

TANSEY, E. A.; ROE, S. M.; JOHNSON, C. D. The sympathetic release test: a test used to assess thermoregulation and autonomic control of blood flow. Advances in Physiology Education, 38, 1, 87-92, 2014.

TRUJILLO C.; ANDERSON T.R.; PELAEZ N.J. A model of how different biology experts explain molecular and cellular mechanisms. Cbe Life Sci Educ, 14: 1 - 15, 2015.

VAN MIL, M.H.W.;BOERWINKEL, D.J.; WAARLO, A.J. Modelling Molecular Mechanisms: A Framework of Scientific Reasoning to Construct Molecular-Level Explanations for Cellular Behaviour. Sci \& Educ, 22: 93-118, 2013.

VERHOEFF, R.; WAARLO, A.; BOERSMA, K. Systems modelling and the development of coherent understanding of cell biology. International Journal of Science Education, 30, 543-568, 2008.

VITT, L.; CALDWELL, J. Herpetology: An Introductory Biology of Amphibians and Reptiles. China: Academic Press, 2013.

WESTBROOK, S. L.; MAREK, E. A cross-age study of student understanding of the concept of homeostasis. Journal of Research in Science Teaching, 29, 1, 51-61, 1992.

\section{NOTAS}

1 Las pruebas saber son evaluaciones aplicadas periódicamente por el Ministerio de Educación Nacional de Colombia a través del ICFES, como seguimiento de calidad del sistema educativo.

2 La referencia en esta evidencia de aprendizaje debería ser a los neurotransmisores y no a las neuronas para mantener la referencia al nivel molecular. En este caso está relacionando hormonas, 
que son moléculas, con neuronas que son células, lo que configura una confusión entre niveles distintos de organización, un problema común en la enseñanza y aprendizaje de la biología, que en estándares sería importante evitar.

3 Producción de calor por procesos metabólicos en respuesta a la temperatura ambiental con el fin de proteger al organismo de la exposición al frío y amortiguar la temperatura corporal frente a las fluctuaciones de temperatura ambiental.

4 Estos fenómenos físicos (Convección, conducción, radiación, evaporación) no son asumidos en el sentido estricto de las acciones o actividades producidas por los entes. Son parte del mecanismo porque constituyen vías físicas que contribuyen con la pérdida o ganancia de energía.

Submetido em 14/02/2019

Aprovado em 21/10/2019

\section{Contato:}

Instituto de Biologia

Rua Barão do Geremoabo, s/n, Campus de Ondina

Universidade Federal da Bahia

CEP 40.170-290 - Salvador, BA - Brasil

\section{Dirección Correspondencia:}

Erika Patricia Daza-Pérez

Calle 23A N. 11 - 04. Barrio Villa Andalucía

Málaga, Santander - Colombia. 